\title{
Heading date 1 (Hd1), an ortholog of Arabidopsis CONSTANS, is a possible target of human selection during domestication to diversify flowering times of cultivated rice
}

\author{
Yasuyuki Takahashi ${ }^{\dagger}$ and Ko Shimamoto* \\ Laboratory of Plant Molecular Genetics, Nara Institute of Science and Technology, \\ 8916-5 Takayama, Ikoma, Nara 630-0101, Japan
}

(Received 9 February 2011, accepted 4 July 2011)

\begin{abstract}
During the domestication of rice (Oryza sativa L.), diversification of flowering time was important in expanding the areas of cultivation. Rice is a facultative short day (SD) plant and requires certain periods of dark to induce flowering. Heading date 1 ( $H d 1$ ), a regulator of the florigen gene $H d 3 a$, is one of the main factors used to generate diversity in flowering. Loss-of-function alleles of $\mathrm{Hd} 1$ are common in cultivated rice and cause the diversity of flowering time. However, it is unclear how these functional nucleotide polymorphisms of $H d 1$ accumulated in the course of evolution. Nucleotide polymorphisms within $H d 1$ and $H d 3 a$ were analyzed in 38 accessions of ancestral wild rice Oryza rufipogon and compared with those of cultivated rice. In contrast to cultivated rice, no nucleotide changes affecting $\mathrm{Hd} 1$ function were found in 38 accessions of wild rice ancestors. No functional changes were found in $H d 3 a$ in either cultivated or ancestral rice. A phylogenetic analysis indicated that evolution of the $H d 1$ alleles may have occurred independently in cultivars descended from various accessions of ancestral rice. The non-functional $H d 1$ alleles found in cultivated rice may be selected during domestication, because they were not found or very rare in wild ancestral rice. In contrast with $H d 3 a$, which has been highly conserved, $H d 1$ may have undergone human selection to diversify the flowering times of rice during domestication or the early stage of the cultivation period.
\end{abstract}

Key words: flowering time, domestication, cereal, natural variation, polymorphism

\section{INTRODUCTION}

Rice is one of the most important cereal crops in the world. The domestication and breeding of rice has occurred during the last 8000 years (Khush, 1997; Doebley et al., 2006). Cultivated rice was originally a tropical plant that evolved from a wild rice species, Oryza rufipogon (Garris et al., 2005; Caicedo et al., 2007). For the origin of cultivated rice, single or multiple domestication events have been proposed. Archaeological studies on ancient rice grains support single domestication event. In former hypothesis, $O$. sativa indica varieties were considered to be domesticated from $O$. rufipogon. Then, O. sativa japonica varieties were developed from

Edited by Koji Murai

* Corresponding author. E-mail: simamoto@bs.naist.jp

$\dagger$ Present address: Max Planck Institute for Plant Breeding

Research, Carl von Linne Weg 10, D-50829 Cologne, Germany

Note: Supplementary materials in this article are at http:// www.jstage.jst.go.jp/browse/ggs indica rice (Oka and Morishima, 1982; Chen, 1999). On the other hand, the latter hypothesis which was supported by several genetic distance studies argues that japonica and indica varieties were independently domesticated from different ancestral wild rice species (Londo et al., 2006; Sweeney and McCouch, 2007b). O. rufipogon grows in tropical and sub-tropical regions of Asia and Oceania. During rice domestication, O. sativa acquired various agriculturally important morphological and physiological traits. These include reductions in grain shattering, yield increases, and changes in plant architecture, seed dormancy, starch quality, and seed size (Kovach et al., 2007; Izawa et al., 2009). Genetic studies on these domestication traits have been performed in rice. For example, $q S H 1$ and sh 4 were found by QTL mapping and related to grain shattering which is one of the well studied domestication traits (Li et al., 2006; Konishi et al., 2006; Lin et al., 2007; Zhang et al., 2009). Rc encodes a bHLH protein which causes the change from red grain to white grain (Sweeney et al., 2006, 2007a). Furthermore, grain 
stricture is also an important domestication trait. GW2, qSW5, and GS3 were known as domestication related genes which improved the grain shape (Song et al., 2007; Shomura et al., 2008; Takano-Kai et al., 2009).

Flowering time was also altered during the domestication process. Diversification of flowering times enabled farmers to expand the growing area (Izawa, 2007; Jung and Muller, 2009). Induction of rice flowering is mainly regulated by day length, and flowering is induced under SD conditions. The genetic pathways that control photoperiodic flowering in rice have been well studied, and a number of genes involved in this regulation are known (Tsuji et al., 2008, 2011; Komiya et al., 2009). OsGI, an ortholog of Arabidopsis GIGANTEA, is under the control of the circadian clock and regulates $H d 1$ expression (Hayama et al., 2002, 2003). Hd1, an ortholog in Arabidopsis CONSTANS, regulates expression of the $H d 3 a$ florigen gene (Yano et al., 2000; Hayama et al., 2002; Izawa et al., 2002). Hd1 is a zinc finger type transcriptional activator carrying the conserved CCT ( $C O$, CO-like, TOC1) domain. The CCT domain functions as a nuclear localization signal and the lack of this domain causes a defect in protein function (Robson et al., 2001). Early heading date 1 (Ehd1) encodes a B-type response regulator that acts independently of $\mathrm{Hd} 1$ as a floral regulator, and its ortholog has not been found in Arabidopsis (Doi et al., 2004). Therefore, Ehd1 is a rice-specific flowering regulator. $H d 1$ and $E h d 1$ independently regulate $H d 3 a$ expression under SD conditions (Tsuji et al., 2008; Komiya et al., 2009). Hd3a is a rice ortholog of Arabidopsis FLOWERING LOCUS T (FT) and encodes a florigen, which is a mobile flowering signal (Kojima et al., 2002; Tamaki et al., 2007; Corbesier et al., 2007; Jaeger and Wigge, 2007; Mathieu et al., 2007). Furthermore, RICE FLOWERING LOCUS T1 (RFT1), which belongs to the $F T$-like gene family, encodes a second floral activator that mainly works under long day (LD) conditions (Komiya et al., 2008, 2009).

We have previously reported on the molecular mechanisms that promote diversity in flowering times in cultivated rice, and showed that $H d 1$ is one of the major determinants of flowering time (Takahashi et al., 2009). Five different types of nucleotide changes that critically affect the protein activity of $\mathrm{Hd} 1$ were found. Nine of the
$17 \mathrm{Hd} 1$ alleles analyzed encoded non-functional $\mathrm{Hd} 1$ proteins. Furthermore, cultivars with non-functional $H d 1$ alleles showed significantly later flowering times than those with functional $H d 1$ alleles (Takahashi et al., 2009). These results suggest that functionally important nucleotide polymorphisms in the $H d 1$ coding region have contributed to the diversity in flowering times in cultivated rice. However, it is not known whether these nucleotide polymorphisms arose before or during domestication. To gain some insight into when these variations in $H d 1$ occurred, we analyzed the nucleotide polymorphisms of $H d 1$ in the progenitor of cultivated rice, O. rufipogon .

\section{MATERIALS AND METHODS}

Plant materials and growth conditions The $O$. rufipogon accessions were retrieved from the Oryzabase (http://www.shigen.nig.ac.jp/rice/oryzabase/top/top.jsp) and supplied by the National Institute of Genetics (Supplementary Table S1). The $O$. sativa core collection was obtained from the National Institute of Agrobiological Sciences Genebank (Kojima et al., 2005; Supplementary Table S2). O. sativa $H d 1$ and $H d 3 a$ alleles used in this study are shown in Takahashi et al. (2009). The plants were grown in climate chambers under short day conditions with daily cycles of $10 \mathrm{~h}$ light at $30^{\circ} \mathrm{C}$ and $14 \mathrm{~h}$ dark at $25^{\circ} \mathrm{C}$. Fluorescent white light (400 to $700 \mathrm{~nm}, 100$ $\mu \mathrm{mol} \mathrm{m}{ }^{-2} \mathrm{~s}^{-1}$ ) was provided at $70 \%$ humidity.

Sequence analysis The coding regions of each gene were amplified using PrimeSTAR HS DNA polymerase (Takara) from genomic DNA, and the PCR products were purified using the MinElute system (QIAGEN). These purified PCR fragments were used as templates, and sequencing reactions were performed using the Big Dye Terminator v3.1 Cycle Sequencing Kit (Applied Biosystems). Data were collected using the ABI PRISM 3100 Genetic Analyzer and analyzed using the GENETYX program ver.7 (GENETYX, Tokyo, Japan).

RNA extraction and cDNA synthesis Leaves were harvested from plants grown under SD conditions 35 days after germination, at the zeitgeber time 16 , which corresponds to the expression peak of $H d 1$. Leaf tissues were

Table 1. Summary of polymorphisms in flowering time genes in rice

\begin{tabular}{|c|c|c|c|c|c|c|c|c|}
\hline \multirow{2}{*}{$\begin{array}{c}\text { Gene } \\
\left(\text { Acc. no. }{ }^{a}\right)\end{array}$} & \multirow{2}{*}{ Species } & \multirow{2}{*}{$\begin{array}{l}\text { No. of } \\
\text { acc. }\end{array}$} & \multicolumn{2}{|c|}{$\mathrm{SNPs}^{\mathrm{b}}$} & \multirow{2}{*}{$\begin{array}{l}\text { Indel } \\
\text { sites }\end{array}$} & \multirow{2}{*}{$\begin{array}{l}\text { F. S. } \\
\text { sites }^{\text {c }}\end{array}$} & \multirow{2}{*}{$\begin{array}{l}\text { Nonsense } \\
\text { mutation }\end{array}$} & \multirow{2}{*}{ No. of alleles } \\
\hline & & & Synonymous & Nonsynonymous & & & & \\
\hline$H d 1$ & O. sativa & 60 & 1 & 13 & 8 & 4 & 1 & 17 \\
\hline$(\mathrm{AB} 041840)$ & O. rufipogon & 38 & 31 & 66 & 17 & 0 & 1 & 33 \\
\hline$H d 3 a$ & O. sativa & 64 & 3 & 4 & 0 & 0 & 0 & 6 \\
\hline (AB052944) & O. rufipogon & 38 & 8 & 12 & 0 & 0 & 0 & 20 \\
\hline
\end{tabular}

\footnotetext{
${ }^{\mathrm{a}}$ GenBank accession number. ${ }^{\mathrm{b}}$ Coding sequence without indel region. ${ }^{\mathrm{c}} \mathrm{F}$. S., frame shift.
} 
ground in liquid nitrogen with the ShakeMaster Auto ver. 2.0 (BioMedicalScience Inc.). Total RNA was extracted using TRIzol (Invitrogen) and treated with DNase I (Invitrogen). cDNA $(20 \mu \mathrm{l})$ was synthesized from $1 \mu \mathrm{g}$ of total RNA using SuperScriptII Reverse Transcriptase (Invitrogen).

Constructs for the luciferase reporter assay The $H d 1$ coding region of each $O$. rufipogon accession was amplified by PCR from cDNA using PrimeSTAR HS DNA polymerase (Takara). Hd1 fragments were subcloned into the $\mathrm{pENTR/D-TOPO}$ vector using the Directional TOPO Cloning kit (Invitrogen). Subsequently, the $H d 1$ was transferred to the CaMV 35S::Gateway cassette plasmid, which contains a pUC12 backbone, by performing an LR recombination reaction (Invitrogen). The $H d 3 a$ promoter sequence was cloned from cv. Nipponbare. This fragment was subcloned into pUC12 along with the luciferase coding region (Photinus pyralis) using the Ligation-Convenience Kit (NIPPON GENE).

Luciferase reporter assays Protoplasts $\left(2 \times 10^{5}\right.$ cells $)$ were prepared from rice Oc cell cultures and transformed using the PEG transfection method (Chen et al., 2006). The cells were transformed with: $1 \mu \mathrm{g}$ of the $p H d 3 a:: p L U C$ construct as a reporter, and $5 \mu \mathrm{g}$ of each $H d 1$ construct containing the $H d 1$ coding sequence driven by the CaMV $35 \mathrm{~S}$ promoter. For a vector control, the bialaphos-resistance gene (Bar) was used instead of the Hd1 coding sequence. After incubation for $12 \mathrm{~h}$, luciferase activities were measured using a Luciferase Assay System (Promega). The luminescence derived from each $H d 1$ allele was normalized for sample protein level and evaluated as a proportion of that of the vector control. Statistical analyses were performed using Microsoft Excel 2003 or Statcel 2 (OMS, Tokyo, Japan).

Phylogenetic analysis The alignment was done using the program GENETYX program ver.7 (GENETYX, Tokyo, Japan) and afterwards inspected carefully and adjusted manually. Phylogenetic reconstruction was done by Neighbor-Joining method (Saitou and Nei, 1987) using the program MEGA5 (Tamura et al., 2011). Bootstrap values were calculated using the same program, number of replicates set to 10,000 (Felsenstein, 1985). The evolutionary distances were calculated using the Maximum Composite Likelihood method (Tamura et al., 2004) and are in the units of the number of base substitutions per site. Gaps were coded as $\mathrm{A} / \mathrm{T}$ matrix at the end of the alignment.

\section{RESULTS}

Nucleotide polymorphisms in the $O$. rufipogon Hd1 coding region We obtained all $38 \mathrm{O}$. rufipogon accessions from 277 accession of wild rice core collection (The

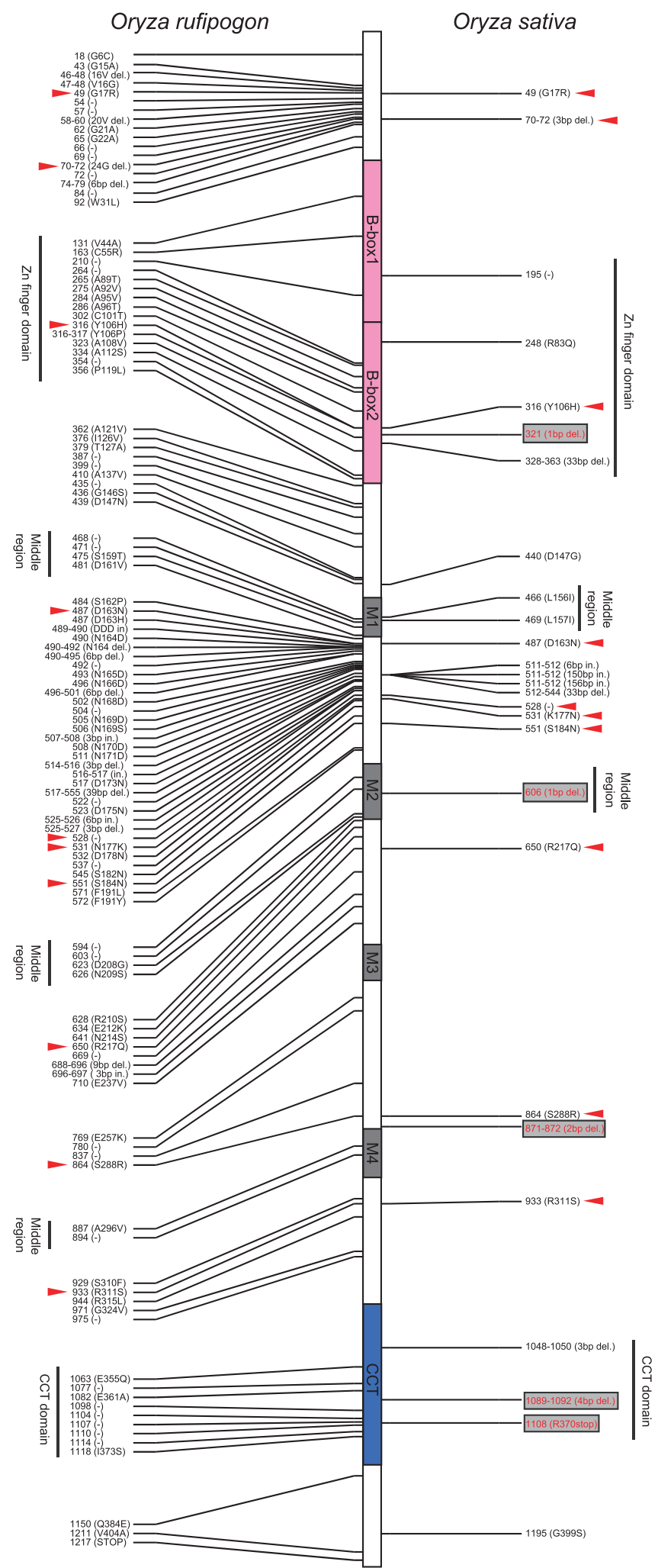

Fig. 1. Comparison of nucleotide polymorphisms within the $H d 1$ coding sequences of $O$. sativa and O. rufipogon. Sites that are polymorphic in nucleotide and amino acid sequences are shown. Red arrowheads indicate the sites of polymorphisms shared by both $O$. sativa and $O$. rufipogon. The locations of nucleotide changes that cause loss of protein function are boxed. 


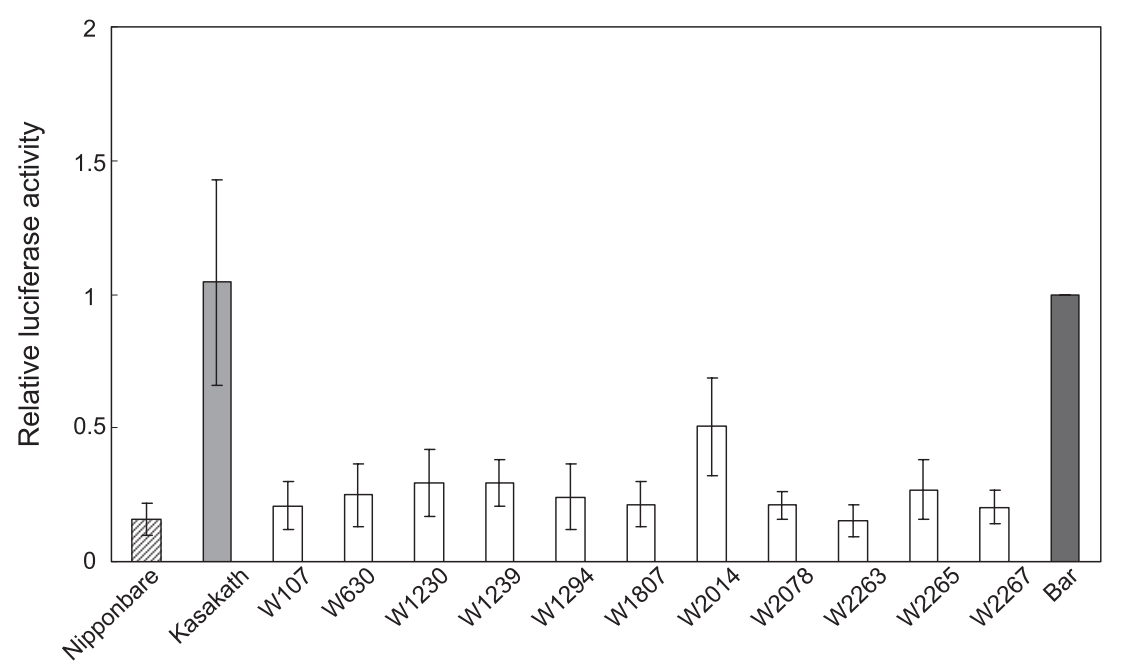

Fig. 2. Activities of $H d 1$ alleles determined using transient luciferase reporter assays in rice protoplasts. The $H d 1$ alleles of cv. Nipponbare and cv. Kasalath were used as functional and nonfunctional controls, respectively. The bialaphos resistance gene (Bar) was used as a vector control. Error bars represent S.D., $n=6$.

National BioResource Project, Japan), which were derived from all over the world and represent the diversity of wild rice accessions in 18 species from 9 genomes covering AA, $\mathrm{BB}, \mathrm{CC}, \mathrm{BBCC}, \mathrm{CCDD}, \mathrm{EE}, \mathrm{FF}, \mathrm{GG}$ and HHJJ. Our sequence analysis identified $33 \mathrm{Hd} 1$ alleles with 97 SNPs among them (Table 1). Among the SNPs, 31 were synonymous and 66 were non-synonymous nucleotide changes. Furthermore, 12 deletions, 5 insertions, and one nonsense mutation were identified, but all the deletions and insertions were in-frame and thus no frameshift mutations were detected (Fig. 1). The one nonsense mutation (position 1217) is at the very end of the coding sequence, and this mutation does not seem to affect protein function. Comparison with the $O$. sativa $H d 1$ alleles revealed 10 identical SNPs and 3 common alleles; the $O$. rufipogon $\mathrm{W} 120, \mathrm{~W} 1852$, and $\mathrm{W} 1715$ alleles are identical to the $O$. sativa Type 1 , Type 4 and Type 5 Hd1 alleles, respectively. Our previous study identified 5 different nucleotide changes in the $H d 1$ coding region of $O$. sativa: four frame-shift and one nonsense mutation, all of which cause defects in the CCT domain (Takahashi et al., 2009). Interestingly, these nucleotide changes were not found in $O$. rufipogon, suggesting that the non-functional $H d 1$ mutations found in cultivated rice may have arisen during domestication.

Functional assays of $O$. rufipogon $\mathrm{Hd} 1$ alleles Although no frame-shift or nonsense mutations were detected in the $H d 1$ gene of $O$. rufipogon, several amino acid substitutions were found in the two conserved regions, the zinc finger and the CCT domains (Fig. 1). Furthermore, 11 other amino acid substitutions were found in other conserved regions. Because it was unclear whether these altered $\mathrm{Hd} 1$ proteins are func-

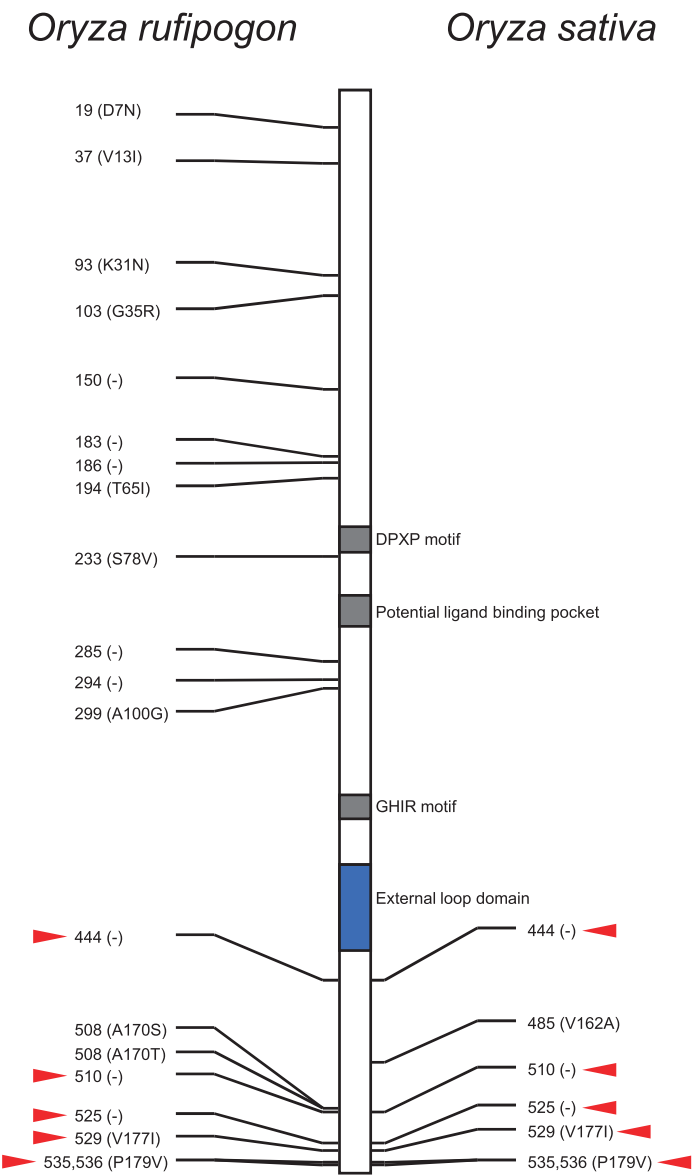

Fig. 3. Comparison of nucleotide polymorphisms within the $H d 3 a$ coding sequences of $O$. sativa and O. rufipogon. Sites that are polymorphic in nucleotide and amino acid sequences are shown. Red arrowheads indicate the sites of polymorphisms shared by both $O$. sativa and $O$. rufipogon. 
tional, we performed transient functional assays in rice protoplasts (Hayama et al., 2002; Takahashi et al., 2009). In this assay, the overexpression of a functional $H d 1$ gene suppresses $H d 3 a$ promoter activity, which is evaluated using the luciferase reporter (Hayama et al., 2002; Takahashi et al., 2009). We examined $11 \mathrm{Hd} 1$ alleles $O$. rufipogon and found that all the alleles examined were functional (Fig. 2). This result clearly demonstrated that $H d 1$ is highly conserved and functional in $O$. rufipogon.

Nucleotide polymorphisms in the O. rufipogon Hd3a coding region Under SD conditions, $H d 1$ regulates expression of the florigen encoding gene (Hayama et al., 2003; Tamaki et al., 2007; Komiya et al., 2009). Therefore, we also investigated the nucleotide polymorphisms in the $H d 3 a$ coding region of $O$. rufipogon. The sequence analysis revealed $20 \mathrm{Hd} 3 a$ alleles (encoding 17 different amino acid sequences) and 20 SNPs among the 38 accessions. Eight SNPs were synonymous, and 12 were non-synonymous nucleotide changes, and no indels were identified (Fig. 3, Table 1). Three alleles are shared between $O$. sativa and $O$. rufipogon: the $O$. rufipogon W630 allele is identical to the $O$. sativa Type 2 allele; the O. rufipogon W1921, W2263, and W2265 alleles

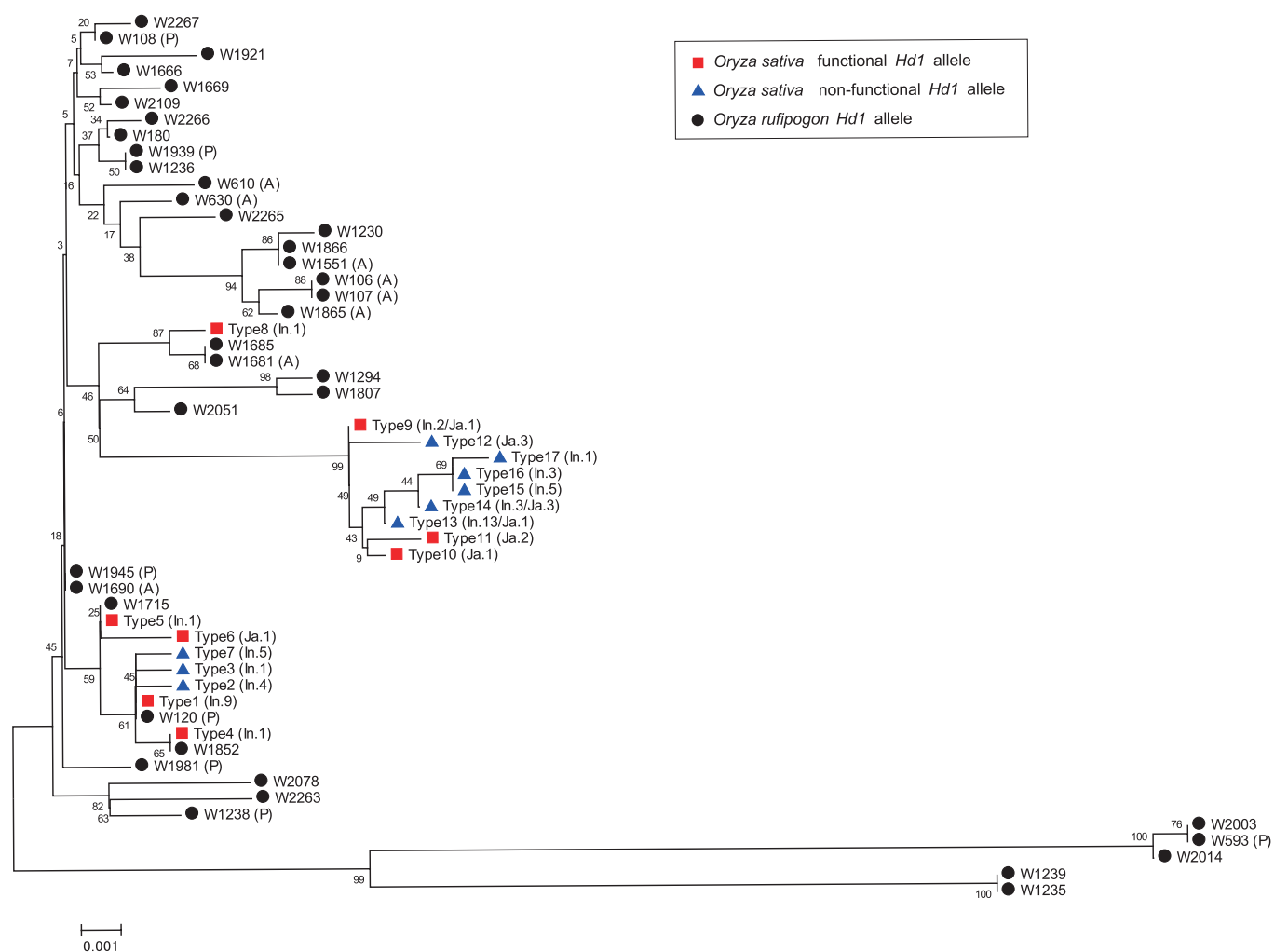

Fig. 4. A phylogenic tree of $H d 1$ alleles from $O$. sativa and $O$. rufipogon. The ecotypes of $O$. rufipogon strains were shown in A, annual; P, perennial. The Hd1 alleles of $O$. sativa are classified as Types 1-17, and the number of each subspecies (In., Indica; Ja., Japonica) with each allele type is given. The percentage of replicate trees in which the associated taxa clustered together in the bootstrap test (10,000 replicates) is shown next to the branches. The scale bar of genetic distance is shown at the bottom. are identical to $O$. sativa Type 3; and $O$. rufipogon W1852 is identical to $O$. sativa Type 6. Mutation sites that have previously been identified in Arabidopsis FT were not altered in the $H d 3 a$ gene of $O$. rufipogon (Pnueli et al., 1998, 2001; Kobayashi et al., 1999; Hanzawa et al., 2005). These results indicate that $\mathrm{Hd} 3 \mathrm{a}$ function is highly conserved in both of the Oryza species. Furthermore, a comparison of $H d 3 a$ sequences in the two species indicated that almost all the nucleotide changes found in $O$. sativa are conserved in $O$. rufipogon, suggesting that very few nucleotide substitutions occurred during domestication. This situation is in strong contrast to that of Hd1 (Fig. 1).

Phylogenetic analysis of $\mathrm{Hd1}$ alleles in $O$. sativa and $O$. rufipogon To understand how various $H d 1$ alleles of $O$. sativa evolved from those in ancestral wild rice, we generated a phylogenetic tree of all the alleles. Several well-separated clusters were identified, and some of these contained alleles from both $O$. sativa and $O$. rufipogon. This suggested that the $H d 1$ alleles of cultivated rice may have evolved independently from multiple $O$. rufipogon accessions. Indeed, three alleles were found in both $O$. sativa and $O$. rufipogon, and the $O$. rufipogon accessions containing these alleles were col-

Oryza sativa non-functional $H d 1$ allele

Oryza rufipogon Hd1 allele 
lected in India (W120), Thailand (W1852), and China (W1715) (Fig. 4, Supplementary Fig. S1). Furthermore, several clusters contained both functional and non-functional $H d 1$ alleles from $O$. sativa. These results suggest that the non-functional $H d 1$ alleles of cultivated rice may have arisen during domestication or the early stage of the cultivation period, and that $H d 1$ may be one of the targets of selection during rice breeding.

\section{DISCUSSION}

Although the $H d 1$ alleles of $O$. sativa are generally less polymorphic than those of $O$. rufipogon, they are functionally more diverse due to the presence of functionally important mutations (Takahashi et al., 2009). Five different types of functionally important mutations have been found in the O. sativa Hd1 gene (Takahashi et al., 2009). Since ca. 8000 years of rice domestication is very short to accumulate these mutations in $O$. sativa it is likely that $H d 1$ has gone through strong positive selection to diversify its function. The very rapid evolution of genes during domestication may be generally caused by high mutation frequency and strong selective advantages of mutations. In the case of rice $H d 1$, mutations having selective advantage are frame-shift and nonsense mutations. Since these mutations appear with much higher frequencies than those with selective advantages this may have caused the unique evolution of rice $H d 1$ during domestication. We also note that the most of $O$. sativa cultivars used in this study are landrace, and no specific mutations of $H d 1$ were observed in modern cultivars (Supplementary Table S2). Therefore, almost all the mutations of Hd1 may not be selected in modern breeding during 20th century but during domestication including the early stage of the cultivation period.

Domestication-related genes have been reported as factors associated with agronomically important traits (Kovach et al., 2007; Izawa et al., 2009). Flowering time genes are also related to domestication since their expression has been modified to expand cultivation areas and to alter various breeding styles (Izawa, 2007; Izawa et al., 2009; Jung and Muller, 2009). For example, the growth of rice plants is strongly affected by temperature; low temperatures during pollen formation cause decreases in pollen fertility. Therefore, to expand rice cultivation to regions of high latitude, the timing of flowering and fertilization must be altered so that they occur before the arrival of the cold season. However, higher latitude regions have longer day lengths in the summer, and this is unfavorable for rice flowering. Hd1 activates flowering under SD conditions, but under LD conditions Hd1 represses flowering (Hayama et al., 2003; Komiya et al., 2009). Therefore, mutations that suppress $H d 1$ activity make rice less sensitive to $\mathrm{SD}$ conditions, and result in rice cultivars that can be grown at higher latitudes. In this sense, Hd1 can be defined as a "diversification" gene rather than a domestication gene (Gross and Olsen, 2010).

We found that functionally important mutations of $H d 1$ emerged independently in various regions of Asia. The fact that $O$. sativa and $O$. rufipogon share several $H d 1$ alleles suggests that various domestic rice cultivars inherited $H d 1$ independently from different accessions of ancestral wild rice. This is consistent with a model of cultivated rice arising through multiple domestications (Kovach et al., 2009). The decreased Hd1 activity may have played a role in expanding the growing area to high latitude regions during the early stages of domestication. On the other hand, there is some overlap in the growth areas of cultivated rice and $O$. rufipogon in tropical areas (Takahashi et al., 2009). This could be explained by the fact that changes in flowering time contribute not only to the expansion of cultivation areas but also to yield. Delayed flowering caused by a decrease in Hd1 activity may result in higher yields. This is also supported by the entangled evolution of the $H d 1$ alleles (Fig. 4). Crossing with other rice strains and human selection may have also accelerated the evolution of diversity in flowering time.

We found that most of the cultivars which possess the same non-functional $H d 1$ alleles are widely distributed in Asian countries (Supplementary Table S2), indicating that the acquisition of non-functional $H d 1$ alleles might have occurred before expansion of the cultivation areas. On the other hand, $H d 1$ allele of W120 (Type $1 H d 1$ allele) is identical to Type 2, 3 and 7 non-functional $H d 1$ alleles except for the frame-shift mutation. Although W120 was collected from India, cultivars carrying Type 2, 3 and 7 non-functional $H d 1$ alleles are derived from Philippines, China, Korea and Myanmar. Therefore, at this moment it is difficult to determine whether these non-functional Hd1 alleles have directly arisen from ancestral wild strains or from the rice cultivars with Type $1 H d 1$ alleles. To answer this question, further study using more detailed geographical information need to be performed.

We notice that the data in this study do not preclude the possibility that some of the non-functional $H d 1$ alleles exist in other $O$. rufipogon accessions that were not examined in this study. Furthermore, in the past, some $O$. rufipogon populations could contain non-functional $H d 1$ alleles under certain ecological conditions which are different from now. At this stage of research, therefore, our conclusions should be treated as a hypothesis that requires further testing with larger scale of wild rice materials and investigation for nucleotide diversity in surrounding genomic region to examine the evidence for a selective sweep. Furthermore, the frequency of many deleterious mutations which was accumulated in the absence of effective recombination during domestication artificial selection has been reported (Lu et al., 2006; Cruz 
et al., 2008). It is clear that defective mutations in $H d 1$ generate the diversity of flowering time in $O$. sativa cultivars (Takahashi et al., 2009), but the detailed investigation should be required to firmly conclude the $\mathrm{Hd} 1$ is a target of human selection during rice domestication.

In this study we focused on the molecular evolution of Hd1 during rice domestication. In the future it will be interesting to investigate how other flowering regulators function in O. rufipogon. Ehd1 is an important regulator of Hd3 expression (Doi et al., 2004), and variations in Ehd1 expression were also shown to contribute to the diversity of flowering time in cultivated rice (Takahashi et al., 2009). Ghd7, which is a negative regulator of Ehd1 and flowering, is another attractive candidate for further investigation (Xue et al., 2008). Furthermore, $R F T 1$, the closest homolog to $H d 3 a$, is a floral regulator under LD conditions (Komiya et al., 2008, 2009). We also showed that cis-regulatory region of $H d 3 a$ is a potential factor generating diversity in flowering time (Takahashi et al., 2009). The analysis of all these flowering time genes will contribute greatly to our understanding of the variations in flowering induction during rice domestication.

\section{CONCLUSIONS}

In this study we examined two key regulators of flowering time in rice: $H d 1$ and $H d 3 a$. We analyzed their evolution during domestication by comparing their nucleotide polymorphisms in $O$. sativa and $O$. rufipogon. Our analysis showed that in contrast with cultivated rice, no functional variations in $H d 1$ were found in 38 accession of wild ancestral rice. Furthermore, a phylogenetic analysis showed that the $H d 1$ alleles in cultivated rice might have evolved at multiple independent locations. Together, these results suggest that the non-functional Hd1 alleles found in cultivated rice originated during domestication. In contrast to $H d 1$, very few nucleotide polymorphisms that affect protein function were found in the $H d 3 a$ florigen-encoding gene of either $O$. sativa or $O$. rufipogon. Decreases in Hd1 activity may have been desirable for the expansion of rice cultivation to higher latitude areas, and in the development of varieties adapted to various environments at multiple locations around the world. Thus, Hd1 may have been a target of human selection to diversify flowering time during rice domestication or the early stage of the cultivation period.

We thank Dr. Hideki Innan (The Graduate University for Advanced Studies) for valuable comments on the manuscript and Dr. Christiane Kiefer (Max Planck Institute for Plant Breeding Research) for technical advice on phylogenetic analysis. The wild rice accessions used in this study were distributed by the National Institute of Genetics, which is supported by the National Bioresource Project, MEXT, Japan. The Oryza sativa core collection was distributed by the NIAS Genebank. This work was supported by Grants-in-Aid for Scientific Research on Priority Areas (grants 10182102 and 19090013 to KS) from the Ministry of Education, Culture, Sports, Science, and Technology of Japan. YT was supported by a fellowship from the Japanese Society for the Promotion of Science.

\section{REFERENCES}

Caicedo, A.L., Williamson, S. H., Hernandez, R. D., Boyko, A., Fledel-Alon, A., York, T. L., Polato, N. R., Olsen, K. M., Nielsen, R., McCouch, S. R., et al. (2007) Genome-wide patterns of nucleotide polymorphism in domesticated rice. PLoS Genet. 3, 1745-1756.

Chen, B. (1999) Origin of 8000-year-old cultivated rice in Henan's Jia Lake site. Agric. Archaeol. 1, 55-57.

Chen, S., Tao, L., Zeng, L., Vega-Sanchez, M. E., Umemura, K., and Wang, G.-L. (2006) A highly efficient transient protoplast system for analyzing defence gene expression and protein-protein interactions in rice. Mol. Plant Pathol. 7, 417427.

Corbesier, L., Vincent, C., Jang, S., Fornara, F., Fan, Q., Searle, I., Giakountis, A., Farrona, S., Gissot, L., Turnbull, C., et al. (2007) FT protein movement contributes to long-distance signaling in floral induction of Arabidopsis. Science 316, 1030-1033.

Cruz, F., Vila, C., and Webster, M. T. (2008) The legacy of domestication: accumulation of deleterious mutations in the dog genome. Mol. Biol. Evol. 25, 2331-2336.

Doebley, J.F., Gaut, B.S., and Smith, B.D. (2006) The molecular genetics of crop domestication. Cell 127, 1309-1321.

Doi, K., Izawa, T., Fuse, T., Yamanouchi, U., Kubo, T., Shimatani, Z., Yano, M., and Yoshimura, A. (2004) Ehd1, a B-type response regulator in rice, confers short-day promotion of flowering and controls FT-like gene expression independently of Hd1. Genes Dev. 18, 926-936.

Felsenstein, J. (1985) Confidence limits on phylogenies: An approach using the bootstrap. Evolution 39, 783-791.

Garris, A. J., Tai, T. H., Coburn, J., Kresovich, S., and McCouch, S. (2005) Genetic structure and diversity in Oryza sativa L. Genetics 169, 1631-1638.

Gross, B. L., and Olsen, K. M. (2010) Genetic perspectives on crop domestication. Trends Plant Sci. 15, 529-537.

Hanzawa, Y., Money, T., and Bradley, D. (2005) A single amino acid converts a repressor to an activator of flowering. Proc. Natl. Acad. Sci. USA 102, 7748-7753.

Hayama, R., Izawa, T., and Shimamoto, K. (2002) Isolation of rice genes possibly involved in the photoperiodic control of flowering by a fluorescent differential display method. Plant Cell Physiol. 43, 494-504.

Hayama, R., Yokoi, S., Tamaki, S., Yano, M., and Shimamoto, K. (2003) Adaptation of photoperiodic control pathways produces short-day flowering in rice. Nature 422, 719-722.

Izawa, T. (2007) Adaptation of flowering-time by natural and artificial selection in Arabidopsis and rice. J. Exp. Bot. 58, 3091-3097.

Izawa, T., Oikawa, T., Sugiyama, N., Tanisaka, T., Yano, M., and Shimamoto, K. (2002) Phytochrome mediates the external light signal to repress FT orthologs in photoperiodic flowering of rice. Genes Dev. 16, 2006-2020.

Izawa, T., Konishi, S., Shomura, A., and Yano, M. (2009) DNA changes tell us about rice domestication. Curr. Opin. Plant Biol. 12, 185-192.

Jaeger, K. E., and Wigge, P. A. (2007) FT protein acts as a longrange signal in Arabidopsis. Curr. Biol. 17, 1050-1054.

Jung, C., and Muller, A. E. (2009) Flowering time control and 
applications in plant breeding. Trends Plant Sci. 14, 563573.

Khush, G. S. (1997) Origin, dispersal, cultivation and variation of rice. Plant Mol. Biol. 35, 25-34.

Kobayashi, Y., Kaya, H., Goto, K., Iwabuchi, M., and Araki, T. (1999) A pair of related genes with antagonistic roles in mediating flowering signals. Science 286, 1960-1962.

Kojima, S., Takahashi, Y., Kobayashi, Y., Monna, L., Sasaki, T., Araki, T., and Yano, M. (2002) Hd3a, a rice ortholog of the Arabidopsis FT gene, promotes transition to flowering downstream of $H d 1$ under short-day conditions. Plant Cell Physiol. 43, 1096-1105.

Kojima, Y., Ebana, K., Fukuoka, S., Nagamine, T., and Kawase, M. (2005) Development of an RFLP-based rice diversity reserch set of germplasm. Breed. Sci. 55, 431-440.

Komiya, R., Ikegami, A., Tamaki, S., Yokoi, S., and Shimamoto, K. (2008) Hd3a and RFT1 are essential for flowering in rice. Development 135, 767-774.

Komiya, R., Yokoi, S., and Shimamoto, K. (2009) A gene network for long-day flowering activates RFT1 encoding a mobile flowering signal in rice. Development 136, 3443-3450.

Konishi, S., Izawa, T., Lin, S. Y., Ebana, K., Fukuta, Y., Sasaki, T., and Yano, M. (2006) An SNP caused loss of seed shattering during rice domestication. Science 312, 1392-1396.

Kovach, M. J., Sweeney, M. T., and McCouch, S. R. (2007) New insights into the history of rice domestication. Trends Genet. 23, 578-587.

Kovach, M. J., Calingacion, M. N., Fitzgerald, M. A., and McCouch, S. R. (2009) The origin and evolution of fragrance in rice (Oryza sativa L.). Proc. Natl. Acad. Sci. USA 106, 14444-14449.

Li, C., Zhou, A., and Sang, T. (2006) Rice domestication by reducing shattering. Science 311, 1936-1939.

Lin, Z., Griffith, M. E., Li, X., Zhu, Z., Tan, L., Fu, Y., Zhang, W., Wang, X., Xie, D., and Sun, C. (2007) Origin of seed shattering in rice (Oryza sativa L.). Planta 226, 11-20.

Londo, J. P., Chiang, Y. C., Hung, K. H., Chiang, T. Y., and Schaal, B. A. (2006) Phylogeography of Asian wild rice, Oryza rufipogon, reveals multiple independent domestications of cultivated rice, Oryza sativa. Proc. Natl. Acad. Sci. USA 103, 9578-9583.

Lu, J., Tang, T., Tang, H., Huang, J., Shi, S., and Wu, C. I. (2006) The accumulation of deleterious mutations in rice genomes: a hypothesis on the cost of domestication. Trends Genet. 22, 126-131.

Mathieu, J., Warthmann, N., Kuttner, F., and Schmid, M. (2007) Export of FT protein from phloem companion cells is sufficient for floral induction in Arabidopsis. Curr. Biol. 17, $1055-1060$.

Oka, H. I., and Morishima, H. (1982) Phylogenetic differentiation of cultivated rice, XXIII. Potentiality of wild progenitors to evolve the Indica and Japonica types of rice cultivars. Euphytica 31, 41-50.

Pnueli, L., Carmel-Goren, L., Hareven, D., Gutfinger, T., Alvarez, J., Ganal, M., Zamir, D., and Lifschitz, E. (1998) The SELF-PRUNING gene of tomato regulates vegetative to reproductive switching of sympodial meristems and is the ortholog of CEN and TFL1. Development 125, 1979-1989.

Pnueli, L., Gutfinger, T., Hareven, D., Ben-Naim, O., Ron, N., Adir, N., and Lifschitz, E. (2001) Tomato SP-interacting proteins define a conserved signaling system that regulates shoot architecture and flowering. Plant Cell 13, 2687-2702.

Robson, F., Costa, M. M., Hepworth, S. R., Vizir, I., Pineiro, M., Reeves, P. H., Putterill, J., and Coupland, G. (2001) Func- tional importance of conserved domains in the floweringtime gene CONSTANS demonstrated by analysis of mutant alleles and transgenic plants. Plant J. 28, 619-631.

Saitou, N., and Nei, M. (1987) The neighbor-joining method: A new method for reconstructing phylogenetic trees. Mol. Biol. Evol. 4, 406-425.

Shomura, A., Izawa, T., Ebana, K., Ebitani, T., Kanegae, H., Konishi, S., and Yano, M. (2008) Deletion in a gene associated with grain size increased yields during rice domestication. Nat. Genet. 40, 1023-1028.

Song, X. J., Huang, W., Shi, M., Zhu, M. Z., and Lin, H. X. (2007) A QTL for rice grain width and weight encodes a previously unknown RING-type E3 ubiquitin ligase. Nat. Genet. 39, 623-630.

Sweeney, M. T., Thomson, M. J., Pfeil, B. E., and McCouch, S. (2006) Caught red-handed: $R c$ encodes a basic helix-loophelix protein conditioning red pericarp in rice. Plant Cell 18, 283-294.

Sweeney, M. T., Thomson, M. J., Cho, Y. G., Park, Y. J., Williamson, S. H., Bustamante, C. D., and McCouch, S. R. (2007a) Global dissemination of a single mutation conferring white pericarp in rice. PLoS Genet. 3, e133.

Sweeney, M., and McCouch, S. (2007b) The complex history of the domestication of rice. Ann. Bot. 100, 951-957.

Takahashi, Y., Teshima, K. M., Yokoi, S., Innan, H., and Shimamoto, K. (2009) Variations in Hd1 proteins, Hd3a promoters, and Ehd1 expression levels contribute to diversity of flowering time in cultivated rice. Proc. Natl. Acad. Sci. USA 106, 4555-4560.

Takano-Kai, N., Jiang, H., Kubo, T., Sweeney, M., Matsumoto, T., Kanamori, H., Padhukasahasram, B., Bustamante, C. Yoshimura, A., Doi, K., et al. (2009) Evolutionary history of $G S 3$, a gene conferring grain length in rice. Genetics $\mathbf{1 8 2}$, 1323-1334.

Tamaki, S., Matsuo, S., Wong, H. L., Yokoi, S., and Shimamoto, $\mathrm{K}$. (2007) Hd3a protein is a mobile flowering signal in rice Science 316, 1033-1036.

Tamura, K., Nei, M., and Kumar, S. (2004) Prospects for inferring very large phylogenies by using the neighbor-joining method. Proc. Natl. Acad. Sci. USA 101, 11030-11035.

Tamura, K., Peterson, D., Peterson, N., Stecher, G., Nei, M., and Kumar, S. (2011) MEGA5: Molecular evolutionary genetic analysis using maximum likelihood, evolutionary distance, and maximum parsimony methods. Mol. Biol. Evol. (in press)

Tsuji, H., Tamaki, S., Komiya, R., and Shimamoto, K. (2008) Florigen and the photoperiodic control of flowering in rice. Rice 1, 25-35.

Tsuji, H., Taoka, K., and Shimamoto, K. (2011) Regulation of flowering in rice: two florigen genes, a complex gene network, and natural variation. Curr. Opin. Plant Biol. 14, $45-52$.

Xue, W., Xing, Y., Weng, X., Zhao, Y., Tang, W., Wang, L., Zhou, H., Yu, S., Xu, C., Li, X., and Zhang, Q. (2008) Natural variation in $G h d 7$ is an important regulator of heading date and yield potential in rice. Nat. Genet. 40, 761-767.

Yano, M., Katayose, Y., Ashikari, M., Yamanouchi, U., Monna, L., Fuse, T., Baba, T., Yamamoto, K., Umehara, Y., Nagamura, Y., et al. (2000) Hd1, a major photoperiod sensitivity quantitative trait locus in rice, is closely related to the Arabidopsis flowering time gene CONSTANS. Plant Cell 12, 2473-2484.

Zhang, L. B., Zhu, Q., Wu, Z. Q., Ross-Ibarra, J., Gaut, B. S., Ge, S., and Sang, T. (2009) Selection on grain shattering genes and rates of rice domestication. New Phytol. 184, 708-720. 